\title{
Visuospatial processing deficits linked to posterior brain regions in premanifest and early stage Huntington's disease
}

\begin{tabular}{|c|c|}
\hline Journal: & Journal of the International Neuropsychological S \\
\hline Manuscript ID & JINS\#-15-RR-206.R3 \\
\hline Manuscript Type: & Regular Research \\
\hline Date Submitted by the Author: & 22-Mar-2016 \\
\hline Complete List of Authors: & $\begin{array}{l}\text { Labuschagne, Izelle; Australian Catholic University, School of Psychology; } \\
\text { Monash University, School of Psychological Sciences } \\
\text { Cassidy, Amy; London School of Hygiene \& Tropical Medicine, Department } \\
\text { of Medical Statistics } \\
\text { Scahill, Rachael; University College London, UCL Institute of Neurology } \\
\text { Johnson, Eileanoir; University College London, UCL Institute of Neurology } \\
\text { Rees, Elin; University College London, UCL Institute of Neurology } \\
\text { O'Regan, Alison; Monash University, School of Psychological Sciences } \\
\text { Queller, Sarah; Queller Consulting, ; Queller Consulting, } \\
\text { Frost, Chris; London School of Hygiene \& Tropical Medicine, Department of } \\
\text { Medical Statistics } \\
\text { Leavitt, Blair; Centre for Molecular Medicine and Therapeutics, Department } \\
\text { of Medical Genetics } \\
\text { Dürr, Alexandra; Hôpital de la Salpêtrière, Department of Genetics and } \\
\text { Cytogenetics, and INSERMUMR S679, APHP, ICM Institute } \\
\text { Roos, Raymund; Leiden University Medical Centre, Neurology } \\
\text { Owen, Gail; University College London, UCL Institute of Neurology } \\
\text { Borowsky, Beth; CHDI Foundation, CHDI Management } \\
\text { Tabrizi, Sarah; University College London, UCL Institute of Neurology } \\
\text { Stout, Julie; Monash University, School of Psychological Sciences; }\end{array}$ \\
\hline MeSH Keywords: & $\begin{array}{l}\text { Neurodegenerative disease, Occipital lobe, Parietal lobe, Cognition, Basal } \\
\text { ganglia, Voxel-based morphometry, Cortical thickness, MRI }\end{array}$ \\
\hline Topic Areas: & Huntington-s Disease, Parietal Lobes, Neuropathology, Cognitive Science \\
\hline
\end{tabular}

\section{SCHOLARONE ${ }^{m}$ \\ Manuscripts}


Dr. Mieke Verfaellie

Action Editor, JINS

iins@cambridge.org

Dear Dr Verfaellie,

RE: JINS\#-15-RR-206.R2 - "Visuospatial processing deficits linked to posterior brain regions in premanifest and early stage Huntington's disease"

Thank you for accepting our manuscript. We are delighted with this news. We have made the requested amendments to the manuscript. Please find our responses below.

Kind regards,

Izelle Labuschagne and Julie Stout

Editor's comments:

1. typos in abstract:

-- correct spelling of precuneus

-- remove comma in "early visuospatial deficits, to functioning"

RESPONSE: These typos have been corrected.

2. The description of the Johnson et al. (2015) findings on p. $6 / 7$ should be clarified:

-- associations between reduced cortical thickness and performance deficits: which tasks does this refer to?

RESPONSE: We now specifically list the six cognitive tasks that were included in the Johnson et al study, and highlight which tasks showed significant associations. Please see pages 5-6.

-- "the other visuospatial tasks" implies multiple tasks but only mental rotation is mentioned.

RESPONSE: This was an error and should be singular, i.e., "the other visual spatial task". However, because of our new edits to the previous comment listing all the specific tasks from the Johnson et al study, we have deleted this sentence altogether as it became redundant.

3. discussion on p. 21: The sentence pertaining to the role of the fusiform gyrus in color integration is confusing at it occurs in the middle of a discussion of regions that are implicated in Mental rotation and not in Map Search. Yet, fusiform gyrus is involved in both tasks.

RESPONSE: We agree that this was out of place and have moved this discussion further down to the next paragraph. Please see our edits on page 20.

4. please correct the following typos:

-- p. 20: "that extend" should be "that extends"

-- p. 23: "is need" should be "is needed"

RESPONSE: These typos have been corrected. 
Labuschagne-Visuospatial Deficits in Huntington's Disease

\title{
Visuospatial processing deficits linked to posterior brain regions in premanifest and early stage Huntington's disease
}

\author{
Izelle Labuschagne ${ }^{1,2}$, Amy Mulick Cassidy ${ }^{3}$, Rachael I. Scahill ${ }^{4}$, Eileanoir B. Johnson ${ }^{4}$, Elin \\ Rees $^{4}$, Alison O’Regan ${ }^{2}$, Sarah Queller ${ }^{5}$, Chris Frost ${ }^{3}$, Blair R. Leavitt ${ }^{6}$, Alexandra Dürr ${ }^{7}$, \\ Raymond $\operatorname{Roos}^{8}$, Gail Owen ${ }^{4}$, Beth Borowsky ${ }^{9}$, Sarah J. Tabrizi ${ }^{4}$, Julie C. Stout ${ }^{2}$ and the TRACK- \\ HD Investigators.
}

1. School of Psychology, Australian Catholic University, Melbourne, Victoria, Australia.

2. School of Psychological Sciences, Monash University, Melbourne, Victoria, Australia.

3. Department of Medical Statistics, London School of Hygiene \& Tropical Medicine, London, United Kingdom.

4. UCL Institute of Neurology, University College London, Unite Kingdom.

5. Queller Consulting, 598 Bella Loop, Dunedin, FL 34698, United States of America.

6. Centre for Molecular Medicine and Therapeutics, Department of Medical Genetics, University of British Columbia, Vancouver, BC, Canada.

7. Department of Genetics and Cytogenetics, and INSERMUMR S679, APHP, ICM Institute, Hôpital de la Salpêtrière, Paris, France.

8. Department of Neurology, Leiden University Medical Centre, Leiden, Netherlands.

9. CHDI Management/CHDI Foundation, Princeton, NJ, USA

Correspondence to: Prof Julie C. Stout

School of Psychological Sciences, Faculty of Medicine, Nursing and Health Sciences, Monash University, Melbourne, Victoria, Australia 3800

Email: julie.stout@monash.edu; Telephone: +61 39905 3987; Fax: +61 399053948 
Labuschagne-Visuospatial Deficits in Huntington's Disease

Word count: Abstract $=250$, Main text $=4999$ 
Labuschagne-Visuospatial Deficits in Huntington's Disease

\begin{abstract}
Objective: Visuospatial processing deficits have been reported in Huntington's disease (HD). To date, no study has examined associations between visuospatial cognition and posterior brain findings in HD. Method: We compared 119 premanifest $(55>$ and $64<10.8$ years to expected disease onset) and 104 early symptomatic (59 stage-1 and 45 stage-2) gene carriers, with 110 controls on visual search and mental rotation performance at baseline and 12 months. In the disease groups we also examined associations between task performance and disease severity, functional capacity and structural brain measures. Results: Cross-sectionally, there were strong differences between all disease groups and controls on visual search, and between diagnosed groups and controls on mental rotation accuracy. Only the premanifest participants close to onset took longer than controls to respond correctly to mental rotation. Visual search negatively correlated with disease burden and motor symptoms in diagnosed individuals, and positively correlated with functional capacity. Mental rotation ('same') was negatively correlated with motor symptoms in stage-2 individuals, and positively correlated with functional capacity. Visual search and mental rotation were associated with parieto-occipital (pre-/cuneus, calcarine, lingual) and temporal (posterior fusiform) volume and cortical thickness. Longitudinally, visual search deteriorated over 12 months in stage- 2 individuals, with no evidence of declines in mental rotation. Conclusion: Our findings provide evidence linking early visuospatial deficits to functioning and posterior cortical dysfunction in HD. The findings are important since large research efforts have focused on fronto-striatal mediated cognitive changes, with little attention given to aspects of cognition outside of these areas.
\end{abstract}

Keywords: Neurodegenerative disease; Occipital lobe; Parietal lobe; Cognition; Basal ganglia; Voxel-based morphometry; Cortical thickness; MRI 
Labuschagne-Visuospatial Deficits in Huntington's Disease

\section{INTRODUCTION}

Huntington's disease (HD) is a debilitating progressive neurodegenerative disease that disrupts all aspects of life due to the broad range of symptoms affecting movement, and psychiatric and cognitive functions. Fronto-striatal damage is a key element of the gross pathological findings in HD (Baizer, Desimone, \& Ungerleider, 1993; Yeterian, \& Pandya, 1995). Consistent with damage to fronto-striatal circuitry, HD is associated with impairments in attention, working memory, processing speed, psychomotor functions, episodic memory, emotion recognition and executive functions (Duff, Beglinger, Theriault, Allison, \& Paulsen, 2010a; Stout et al., 2011; Labuschagne et al., 2013). A growing body of research suggests that HD also affects visuospatial processing (Mohr et al., 1991; Lawrence, Watkins, Sahakian, Hodges, \& Robbins, 2000; Lineweaver, Salmon, Bondi, \& Corey-Bloom, 2005). Posterior cortical areas, including the parietal and occipital cortices which subserve visuospatial functions, also show structural changes in HD, including volume loss, reduced cortical thickness, and associated white-matter abnormalities (Tabrizi et al., 2009). In addition, changes in brain metabolites (NNA and glutamate) in the posterior cingulate have also been reported in HD (Unschuld et al., 2012). Although decline in visuospatial performance has not been directly associated with posterior cortical brain changes in HD, these deficits are hypothesised to be linked to occipital and parietal degeneration.

Deficits in visuospatial performance have been demonstrated across a variety of cognitive tasks in HD (Gomez-Tortosa, del Barrio, Barroso, \& Garcia Ruiz, 1996; Lawrence et al., 2000; Lineweaver et al., 2005), but it is the ability to spatially manipulate information that is particularly poor in HD and tends to worsen with disease progression (Mohr et al., 1991). This finding points to the possible role in the dorsal visual stream in HD pathology. The dorsal stream is one of two visual pathways in primates known to be important for spatial perception and localisation (BaronCohen, Wheelwright, Hill, Raste, \& Plumb, 2001). The dorsal stream involves projections from the visual cortex to the anterior parietal lobe where information is integrated about where objects 
are, and provides essential input that directs movement in relation to objects in space (Goodale, \& Milner, 1992; Kravitz, Saleem, Baker, \& Mishkin, 2011; Mandal, Joshi, \& Saharan, 2012). Brain lesion, neuroimaging, and brain stimulation studies indicate that the parietal lobes play an important function in tasks involving mental rotation and visual search (Harris et al., 2000; Donner, Kettermann, Diesch, Villringer, \& Brandt, 2003; Blankenburg et al., 2010). Consistent with the links between 'where' information is and motor planning, functional magnetic resonance imaging (MRI) studies of mental rotation demonstrate activations of the primary motor and premotor cortices (Evans, \& Rothbart, 2007; Rubin et al., 2013), and the subcortical motor system involving the basal ganglia (Harris, Harris, \& Caine, 2002). These foregoing findings support a role for the dorsal visual pathway, motor cortex and basal ganglia in visuospatial functions, and are regions also implicated in the neuropathology of HD.

Given the evidence of visuospatial dysfunction, together with the identification of brain changes in regions known to be important for visuospatial processing in $\mathrm{HD}$, deficits in complex visual processing have been suggested to be markers of disease progression in HD (GomezTortosa et al., 1996). Evidence is also accumulating that visuospatial deficits can be identified in premanifest HD many years before onset (Robins Wahlin, Lundin, \& Dear, 2007; Duff et al., 2010a; Duff et al., 2010b), suggesting that visuospatial abilities may be one of the early cognitive functions to decline in HD. Nonetheless, to date, little is known about the association between posterior brain activity and visuospatial cognition in HD.

Recently, in an attempt to broaden our understanding of the potential posterior cortical involvement in the neuropathology of HD, we examined associations between cortical thickness across specifically the occipital lobe and several cognitive tasks using data from the larger TRACK-HD study (Johnson et al., 2015). These associations between occipital lobe and cognition were examined for six cognitive tasks including Stroop, Trial Making, Symbol Digit, Spot the Change, and two visuospatial tasks Map Search and Mental Rotation. The findings showed 
regionally-specific associations between reduced cortical thickness and performance deficits on five out of six tasks, mostly strongly in the lateral occipital and lingual regions, and no evidence of cuneus involvement. Mental Rotation was the only task that showed no associations with occipital brain regions. Relating to visuospatial function, occipital regions involving the lingual and lateral occipital cortex showed strong associations to a visual search task, Map Search; the other vistospatial tasks, Mental Rotation, showed no associations with posterior brain regions. However, $€$ The focus of Johnson et al. was on the occipital lobe and a range of cognitive tasks for which only a single variable was analysed. Thus, to obtain a clear picture of the nature of the visuospatial deficits in HD, a more detailed investigation, spanning across the posterior brain from occipital lobe to motor regions and across the spectrum of HD, is needed.

Using data from the TRACK-HD longitudinal study, we examined the link between visuospatial processing and structural brain changes in premanifest and early stage diagnosed HD, using two visuospatial tasks: visual search and attention task requiring locating symbols on a map, and a mental rotation task of complex stimuli. Based on previous evidence in healthy participants, we selected tasks that reliably recruit posterior brain regions known to be affected in HD, in particular the occipital lobes, inferior and superior parietal areas and the basal ganglia. We build on our previous work (Johnson et al., 2015) by examining visual search and mental rotation performance at two time points, 12 months apart, and studied associations between task performance and disease severity as well as MRI measures including regional grey- and whitematter volume and cortical thickness. We hypothesised that visuospatial deficits would: be detectable across the HD spectrum, deteriorate over 12 months, correlate with disease severity and functional capacity, and be associated with posterior and striatal brain volumes and cortical thickness.

\section{METHOD}


Labuschagne-Visuospatial Deficits in Huntington's Disease

\section{Participants}

We recruited 104 participants with early HD, 119 HD gene-expanded ( $\geq 40$ CAG repeats) premanifest HD participants, and 110 healthy controls from Paris, Leiden, Vancouver and London for the TRACK-HD study (Tabrizi et al., 2009). Selection criteria for premanifest HD involved Total Motor Scores $\leq 5$, as assessed by the Unified Huntington's Disease Rating Scale (UHDRS) (Huntington Study Group, 1999), and Disease Burden Score $\geq 250$, calculated by age $\mathrm{x}$ [CAG35.5] (Penney, Vonsattel, MacDonald, Gusella, \& Myers, 1997). The premanifest HD participants were divided, based on the baseline group medium (10.8 years) for predicted years to diagnosis, into those far from diagnosis (PreA) and those near diagnosis (PreB). The manifest HD group was divided, as defined by the UHDRS Total Functional Capacity score, into those with stage-1 HD (HD1; scores of 11-13) and stage-2 HD (HD2; scores of 7-10) (Shoulson, \& Fahn, 1979).

Controls, including primarily spouses or partners and gene negative siblings, were frequencymatched on age, sex and education (Table 1). Study entry criteria included ages 18-65 years, ability to tolerate and safely undergo MRI, not participating in a clinical drug trial, and free of other major neurological, psychiatric or medical illnesses, including significant head injury and drug or alcohol abuse. All participants were tested annually as part of the larger TRACK-HD study. It is important to note that the visuospatial assessments reported here were introduced at 24month time point, and were therefore assessed at 24 and 36 months of the TRACK-HD study. However, for simplicity, we have referred to baseline and 12-month follow-up in the current study. Written informed consent was obtained from participants according to the Declaration of Helsinki, and the study was approved by local ethics committees.

\section{[INSERT TABLE 1 HERE]}

\section{Visuospatial assessment}

Map Search 
The Map Search task is a subtest from the Test of Everyday Attention (Robertson, Ward, Ridgeway, \& Nimmo-Smith, 1994), and measures visuospatial selective attention. For the task, participants were timed for 2 minutes while they searched for, and used a pen to circle, a target symbol that occurred in multiple places on a map among other distracter symbols. The map was visually cluttered, similar to any road map, and displayed a portion of the city of Philadelphia in the United States. Participants began performing the task using a pen of a given colour, and then after one minute, the examiner exchanged the pen for a different colour to differentiate responses made in the first and second minutes of testing. We measured the number of correctly circled targets, scored separately at 1 and 2 minutes. The maximum score was 80 . Previous evidence using this task in early HD ( $\mathrm{n}=10)$ vs. controls demonstrated a large effect size of -2.92 (Murray, $\&$ Stout, 1999). We highlight here that we have previously reported on the number correct for 2 minutes using different statistical methods in Johnson et al. (2015).

\section{Mental Rotation}

The computerised Mental Rotation task assesses the ability to mentally rotate 3-dimensional stimuli consisting of images of cubes, attached to each other by sharing a common side or sides (Shepard, \& Metzler, 1971; Peters, \& Battista, 2008). On each trial, a pair of stimuli was presented. One figure in the pair was either a rotation of the other figure, or a rotation of the mirror image of the other figure. Participants had to indicate whether the rotated figure was identical to ("same") or a mirror image of ("mirror") the comparison figure. The stimuli were presented using six degrees of rotation across four difficulty levels: i) 5 degrees, ii) 65 and 305 degrees, iii) 125 and 245 degrees, and iv) 185 degrees. We collapsed the data across degrees that were similar in difficulty, i.e., those degrees that were horizontally opposites and thus required the same amount of rotation from 0 (e.g., 65 and 305 degrees) (Shepard, \& Metzler, 1971; Bethel-Fox, \& Shepard, 1988; Lineweaver et al., 2005). Thus, for simplicity, we subsequently refer to the four angles as 5 , 65 (e.g., 65 and 305, both equal distance from 0), 125 (e.g., 125 and 245, both equal distance from 
0), and 185 degrees. There were ten practice trials, followed by 48 experimental trials comprised of 12 trials each at the four difficulty levels, balanced for SAME and MIRROR conditions. We measured accuracy (percent correct), response time (for correctly identified targets only) and speed-accuracy trade-off (correlation between accuracy and response times). Previous research showed an effect size of -0.68 in premanifest HD $(n=11)$ vs. controls (Robins Wahlin et al., 2007). We highlight here that we previously reported on a total score involving number correct collapsed across all degrees of rotation in a different set of analyses (Johnson et al., 2015).

\section{MRI acquisition}

Participants underwent 3 Tesla MRI scanning, with data acquisition procedures that were standardised according to the TRACK-HD study protocol (Tabrizi et al., 2009). Grey- and whitematter were assessed using voxel-based morphometry (VBM) and cortical thickness using Freesurfer version 5.3; for more details see (Tabrizi et al., 2009).

\section{Statistical analyses}

For Map Search, we separately analysed the number of correct objects in 1 and 2 minutes. To jointly model the proportions at baseline and follow-up, we used a generalised estimating equations (GEE) approach with a logit link, a "working" assumption of independence between the baseline and follow-up, and robust Huber-White standard errors (Huber, 1967) that allow both for the fact that the baseline and follow-up measures are not independent, and that the number of objects identified at each visit does not truly follow a binomial distribution. For Mental Rotation, we separately analysed accuracy and response times as continuous variables, using a mixed-effects regression model with random subject-specific intercepts to simultaneously model the multiple outcomes. We again used robust Huber-White standard errors, which are robust to the residual heteroscedasticity that may be expected between disease groups. All analyses included an effect of time (allowing for the fact that visits were not always exactly one year apart) and adjusted for age, sex, educational level and study site. Effects were permitted to vary with time and, with Mental 
Rotation outcomes, by degree (5/65/125/185 degrees) and condition (SAME/MIRROR). We report between-group comparisons on only the 65 and 125 degree angles because inspection of the data indicated ceiling effects for accuracy at 5 degrees (all participants performed near the 90$100 \%$ accuracy level), and floor effects for accuracy at 185 degrees (the HD groups performed way below chance level); Figure 1.

For cross-sectional comparisons between-groups at baseline, we calculated standardised marginal contrasts between controls and each of the gene-positive groups (PreA, PreB, HD1 and HD2) for each outcome from the models described above. These contrasts compared the expected mean outcomes between groups, after standardising the groups to have the same distribution of adjustment covariates, thus removing the effect of these potential confounders from the contrast (Muller, \& MacLehose, 2014). The expected outcomes were predicted by multiplying probabilities predicted from the GEE (for Map Search) by the number of possible events (here number of targets to be found $=80$ ) or from the mixed model (for Mental Rotation). For longitudinal analyses, we compared annualised change in performance from baseline to 12 months between groups. These standardised contrasts calculated the expected change in the outcome by taking the first derivative of the regression equation with respect to time (in units of one year), calculating in each group the predicted change in the probability (GEE) multiplied by 80 (Map Search) or the outcome (mixed effect model, Mental Rotation), and contrasting the changes. Standard errors of both sets of contrasts were calculated using the delta method (Oehlert, 1992).

For Mental Rotation, we also examined the speed-accuracy trade-off relationship by adding accuracy as a second exposure of interest to the response time regression model. The effect of accuracy in each group was allowed to differ by time, degree of rotation (65 and 125 degrees) and SAME/MIRROR condition. Standardised contrasts using methods described above were used to estimate the effect of accuracy on time taken to correctly identify objects between subgroups at baseline. 
In the gene-positive groups, we then used partial Pearson correlation coefficients adjusted for age, sex, site, education and CAG length to determine how performance accuracy on the visuospatial tasks related to disease severity, measured by the Disease Burden Score (Penney et al., 1997), and Total Functional Capacity and Total Motor Scores taken from the UHDRS. For Disease Burden, we report partial correlations separately for each of the four groups. For Total Motor Score, only the two diagnosed groups are included because in the premanifest group, motor scores are very low and have little variance. Total Functional Capacity is reported for the diagnosed group as a whole because it also has low variance and it serves as the defining difference between disease stages in the two diagnosed groups. Stata v13 (StataCorp, College Station, TX, USA) was used for all statistical analyses involving task performance and disease severity. Because each cognitive task has its own independent interest, no adjustments were made to $p$-values to allow for multiple comparisons (Rothman, 1990).

Finally, we investigated associations between regional brain volume (VBM), and the selected measures (i.e., accuracy) from the Map Search and Mental Rotation (65 and 125 degree) tasks using the combined gene-positive group (PreA, PreB, HD1, HD2), adjusting for age, gender, site, education, intracranial volume, CAG and Disease Burden. Results are corrected for multiple comparisons using a 5\% false discovery rate (Deboer et al., 2013). Likewise, using the combined gene-positive group, associations between cortical thickness and accuracy measures from both tasks were examined using a general linear model and the FreeSurfer 'Different Onset, Same Slope' model (Fischl, \& Dale, 2000; Fischl et al., 2002; Fischl et al., 2004). The model constrained the slopes of any continuous variables to evolve at the same rate in all groups. A DOSS model was deemed appropriate after first investigating that there were no age-related differences on cortical thickness within the groups, the genders or the scanners. A Monte Carlo correction for multiple comparisons was applied (Hagler, Saygin, \& Sereno, 2006), with a vertexwise threshold (cluster forming threshold) of $p<0.05$ (two-tailed) and a cluster-wise threshold of 
$p \mathrm{cw}<0.025$ (i.e., $p \mathrm{cw}<0.05$ corrected across two hemispheres). Results were adjusted for age, gender, site, education, CAG and Disease Burden.

\section{[INSERT FIGURE 1 HERE]}

\section{RESULTS}

\section{Cross-sectional and longitudinal visuospatial performance}

Map Search accuracy

Cross-sectionally at baseline and 12 months, groups of increasing severity of disease showed worse performances (i.e., fewer correct targets found) at both 1 and 2 minutes (Figure 2).

Between-group comparisons at 1-minute showed strong evidence (all $p<0.002$ ) of impaired performance in all disease groups compared to controls, with the differences from controls ranging from 6.3 correct targets $(95 \%$ CI $2.5,10.1)$ for PreA to 22.7 correct targets $(19.1,26.2)$ for HD2; Table 2. The 2-minute scores also showed strong evidence of impaired performance in PreB, HD1, and HD2 $(p<0.0001)$ and a trend for statistical significance in PreA $(p=0.06)$. Longitudinally, there were significant 12-month declines in Map Search performance at both 1 and 2 minutes for the HD2 group relative to controls $[-3.4(-5.8,-1.1) p=0.0043$, and $-3.8(-7.0,-0.7) p=0.0167]$. No other between-group comparisons showed significant differences in annualised change.

\section{[INSERT FIGURE 2 HERE]}

\section{Mental Rotation accuracy}

Cross-sectionally at baseline and 12 months, and for both the SAME and MIRROR conditions, accuracy was typically lower with larger degrees of rotation in all disease groups and controls

(Figure 1, A). Furthermore, the more severe the disease stage the poorer performance tended to be across all conditions (Figure 1, A). There was strong evidence of impairment in the HD1 group on both 65 and 125 degrees for MIRROR trials [-17.0\% (-25.0,-9.0) and -17.5\% (-25.7,-9.3); both $p<0.0001$, and in the HD2 group on both degrees for both SAME and MIRROR trials [ranging 
from $-18.1 \%(-27.9,-8.4)$ to $-11.0 \%(-20.7,-1.3)$; all $p<0.03]$. However, the premanifest HD groups' accuracy appeared similar to that of controls at both degrees. Longitudinally, there was no compelling evidence to suggest that the HD groups changed or declined more rapidly than controls. Four out of the sixteen comparisons made with controls reached formal statistical significance, but one of these was in the direction of reduced changes, and taken as a whole, no clear coherent pattern emerged from these results; Table 2.

\section{[INSERT TABLE 2 HERE]}

\section{Mental Rotation response time}

Cross-sectionally at baseline and 12 months follow-up, in the control and PreA groups the mean response time for correct answers generally increased at each increment in the degree of difficulty under the SAME condition, but stabilised or decreased at the larger degrees in the PreB, HD1 and HD2 groups (Figure 1B). A similar pattern was present under the MIRROR condition except that all groups' mean response times stabilised or decreased at the larger degrees. There were no significant differences in response time at either condition or either 65 and 125 degree between controls and the PreA, HD1 and HD2 groups, but the PreB group was significantly slower than controls at both degrees for both conditions, where differences ranged from $1020 \mathrm{~ms}(343,1698)$ to $937 \mathrm{~ms}(451,1424)$, all $p<0.004$; Table 2. Longitudinally, there was no strong evidence of changes in performance over 12 months in the disease groups compared to controls. Two out of the sixteen comparisons made approached formal statistical significance $(p=0.053$ and $p=0.075)$ but there was no coherent pattern to these results.

\section{Mental Rotation speed-accuracy}

For all groups except PreA, response time for accurate answers tended to be faster in participants with better overall performance. In contrast, PreA participants who performed better tended to be slower when making correct responses (Figure 3). Of the sixteen comparisons made, one comparison, which was between PreA and controls at 65/MIRROR, was statistically significant 
$(p=0.048)$, where for each $10 \%$ absolute increase in accuracy, the difference in the corresponding change in response time between PreAs and controls was 239 ms (95\% CI: 3, 476);

Supplementary Table 1. No other comparisons approached statistical significance, so interpretation of this result must be made with caution.

\section{[INSERT FIGURE 3 HERE]}

\section{Associations between cross-sectional visuospatial accuracy and disease severity}

In HD1, performance on the Map Search 1-minute variable was negatively correlated with Disease Burden (partial $\mathrm{r}=-0.31, p=0.032$ ) and Total Motor Score (partial $\mathrm{r}=-0.30, p=0.041$ ). In HD2, performance on Map Search 1-minute, and accuracy on the Mental Rotation under the SAME condition, were both negatively correlated with Total Motor Score (partial $\mathrm{r}=-0.37, p=0.023$ and partial $\mathrm{r}=-0.35, p=0.034$, respectively). In the combined diagnosed HD group, Total Functional Capacity was associated with both Map Search 1-minute performance (partial $\mathrm{r}=0.38, p<0.001$ ) and Mental Rotation (SAME) accuracy (partial $\mathrm{r}=0.29, p=0.005$ ); Table 3. Disease Burden in the premanifest groups was not associated with visuospatial task performance.

[INSERT TALBE 3 HERE]

\section{Associations between cross-sectional visuospatial accuracy and structural brain}

\section{abnormalities}

Baseline brain volumes, assessed using VBM, were significantly correlated with both Map Search and Mental Rotation tasks, i.e., reduced volume with poorer performance (Figure 4). Specifically, poorer performance (lower scores) on Map Search 1-minute scores correlated with grey-matter volume loss, predominantly in striatal regions of the basal ganglia (caudate, putamen) and to a lesser extend with parieto-occipital regions (precuneus/cuneus, left lingual gyrus), motor areas (precentral gyri), fusiform and insula. Poorer Map Search 1-minute scores were also associated with widespread white-matter volume loss, in the cortiocostriatal tract, cingulum, and cerebellum. 
For Mental Rotation, at 65 degrees and SAME/MIRROR conditions, poorer accuracy was associated with grey-matter volume loss in the striatal regions (caudate, putamen), and also across several cortical regions including parieto-occipital cortex (precuneus, calcarine, lingual regions), motor regions (supplementary motor area, precentral gyrus, cerebellum), temporal lobe (fusiform, middle temporal gyri, temporal pole, hippocampus, parahippocampal region), superior frontal lobe, and cingulate. When SAME and MIRROR conditions were separated, only the MIRROR condition showed significant grey-matter correlations (caudate, supplementary motor area, precentral gyrus, cingulate, temporal pole, cerebellum). Thus, the MIRROR condition appeared to account for most of the grey-matter associations observed in the combined SAME/MIRROR conditions. Poorer accuracy on the combined SAME/MIRROR conditions correlated with whitematter volume loss throughout the brain, but particularly in the striatal region, corpus callosum, brain stem and cerebellum. When the SAME and MIRROR conditions were examined separately, only the SAME condition showed significant white-matter correlations and mainly in the occipital lobe, corticostriatal tract, cingulum, brain stem and cerebellum. Thus, the SAME condition likely accounted for most the white-matter associations observed in the combined SAME/MIRROR conditions.

Adding to these VBM results, poorer 1-minute Map Search scores were associated with significantly less cortical thickness in the superior parietal lobe region extending into the lateral occipital region, $p=0.007$ (Figure 4). For Mental Rotation at 65 degrees and combined SAME/MIRROR conditions, poorer performance was associated with less thickness in the fusiform/lingual region, $p=0.01$ (Figure 4).

[INSERT FIGURE 4 HERE]

\section{DISCUSSION}


This study aimed to elucidate the visuospatial abnormalities across the spectrum of HD using a large sample of subjects. We have several key findings. First, cross-sectionally, we found large effects for comparisons between controls and all premanifest and early HD groups on Map Search, thus demonstrating the sensitivity of the Map Search task for both premanifest and diagnosed HD. In contrast, Mental Rotation performance differed from controls only for the early HD groups, suggesting that compared to Map Search, Mental Rotation, at least in the version we used, was not as sensitive for HD. Second, for Mental Rotation, correct response times were slower in the premanifest near to onset group compared to controls, but not in the premanifest far from onset or diagnosed groups, suggesting the presence of compensatory strategies. Third, for Map Search only, performance deterioration was significant over 12 months in the early HD2 group (more advanced but still relatively early diagnosed HD) but not in premanifest or HD1 groups, suggesting a detectable decline over one year in Map Search at the more severe disease stages. Fourth, in diagnosed individuals, Map Search, but not Mental Rotation, was significantly associated with Disease Burden, motor symptoms and functional capacity in most comparisons to controls. We found no associations between Disease Burden and Map Search or Mental Rotation in the premanifest groups. Finally, we demonstrated correlations between visuospatial performance (Map Search and Mental Rotation) and both brain volume and cortical thickness evident in parieto-occipital (pre-/cuneus, calcarine, lingual) regions, as well as motor and fusiform gyrus regions, suggesting a link between structure and function, thereby substantiating the link between visuospatial performance and posterior brain dysfunction in HD.

\section{Possible compensation during Mental Rotation}

Interestingly, cross-sectional analyses of Mental Rotation response times, showed that those premanifest HD near to onset (PreB) slowed their response times (i.e., took longer to respond) compared to controls. Coupled with the fact that there were no performance differences in the PreB group, whereas the diagnosed groups were less accurate than controls, this may indicate that 
PreB maintained their performance on visual cognitive function by compensating with slower responses, whereas early HD maintained their response times but showed significantly poorer performance accuracy. In our speed-accuracy trade-off results, there was no strong evidence for a difference in the trade-off relationship between any of our gene-positive groups and controls, although there was a weakly significant difference in those premanifest HD far from onset (PreA). PreA participants who performed more accurately overall, took more time to do so; corresponding control participants took slightly less time, giving a total difference of 239 milliseconds per 10\% improvement in accuracy. Previous evidence reported speed-accuracy trade-offs during mental rotation in diagnosed HD (Lineweaver et al., 2005), although the sample size was small and no premanifest HD participants were included. The lack of generality of the speed-accuracy trade-off in Mental Rotation across the HD groups may indirectly suggest that visuospatial compensation processes are evident in the early disease stages, demonstrated by maintained accuracy in the face of slower response times (in PreB), but that these compensation processes are diminished by the time the disease progresses through to the clinical stages. This finding is in line with recent suggestions of compensatory mechanisms in posterior (parietal) brain regions, which are regions implicated in visuospatial processes, in those premanifest HD closest to motor disease onset (Klöppel et al., 2015).

\section{Visual search performance dysfunction occurs early and deteriorates over 12 months}

Performance on the Map Search in all gene-positive groups was worse than controls, including the far from onset PreA group, whereas performance accuracy on the Mental Rotation was worse only in the diagnosed groups. Our findings are in line with previous evidence of visuospatial deficits in HD (Mohr et al., 1991; Gomez-Tortosa et al., 1996; Lawrence et al., 2000; Lineweaver et al., 2005) including in premanifest stages (Robins Wahlin et al., 2007; Duff et al., 2010a; Duff et al., 2010b). Our finding that Map Search showed deficits even in the premanifest stages of HD, suggests that a visual attentional task may be a more sensitive visuospatial marker of disease 
progression than a more complex visuospatial task involving the ability to spatially manipulate information, such as mental rotation. Map Search taps into the everyday behaviour of visually searching for items on a map or other cluttered visual field, and thus may have some ecologicallyrelevant implications. Furthermore, Map Search deteriorated over 12 months in the HD2 group (vs. controls), whereas there was no strong evidence of longitudinal changes on Mental Rotation. It is possible that Map Search, because of its attentional component, taps more into disease-related circuits than Mental Rotation that may contribute to its sensitivity in premanifest HD. However, this remains to be examined in more detailed using larger visual processing protocols.

Nonetheless, this is the first study reporting longitudinal change, although only over 12 months, in visuospatial abilities in HD. Although a relatively short interval, this provides initial evidence of significant and rapid declines in visuospatial/visuo-atttentional processes.

\section{Visuospatial performance correlates with disease severity}

Our findings showed that greater disease severity (disease burden and motor signs) is associated with worse performance on Map Search and Mental Rotation tasks in diagnosed individuals, and that better functional capacity is associated with better performance. This supports our predictions of posterior brain region involvement, as measured by visuospatial performance, in the neuropathology of HD. No previous study has directly linked visuospatial performance to disease severity or functional capacity in HD. For Alzheimer's disease, not only have such links been found, but furthermore, visuospatial functions were most strongly associated with functional ability compared to any other cognitive domain that was examined (Perry, \& Hodges, 2000). These authors also found that visuospatial function was the sole cognitive predictor of functional abilities. These findings suggest the possibility of a link between visuospatial deficits and disease severity as well as functional outcomes in HD. The finding of functional significance may also have implications for clinical trials investigating drug treatments for HD. However, we are 
cautious interpreting these findings as it could merely reflect that visuospatial performance track with the progression of the disease.

\section{Visuospatial performance correlates with posterior cortical and striatal areas}

We found a strong link between visuospatial performance and brain volume in HD, predominantly in occipito-parietal (pre-/cuneus, calcarine, lingual), motor (supplementary motor area, precentral gyrus, cerebellum), and temporal (fusiform) regions. Similarly, our cortical thickness correlations showed that Map Search was associated with thickness of the superior parietal cortex, extending into lateral occipital lobe, and Mental Rotation was associated with thickness of the occipital lobe, extending into the posterior fusiform gyrus. These findings are consistent with prior suggestions of cortico-striatal circuitry with connections that span not only the frontal cortex but also temporal and posterior cortical regions including visual and parietal cortices (Baizer et al., 1993; Yeterian, \& Pandya, 1995). Our findings extend our previous work (Johnson et al., 2015), by showing associations between visuospatial performance involving Map Search and Mental Rotation performances and posterior parts of brain that extend beyond that of the occipital lobe. The findings therefore support our predictions of additional posterior cortical involvement in the neuropathology of HD.

To date, there is very limited evidence linking specific brain measures with visuospatial performance in HD. However, our findings of associations between occipito-parietal and striatal volumetrics and visuospatial processing in $\mathrm{HD}$, are consistent with evidence of dorsal visual stream involvement in visuospatial processing in healthy participants (Harris et al., 2000; Harris et al., 2002; Donner et al., 2003). Dorsal visual stream deficits relating to mental rotation have been implicated in Alzheimer's disease (Prvulovic et al., 2002; Jacobs et al., 2012; Mandal et al., 2012). Our findings may therefore suggest that the dorsal visual pathway could be more susceptible to early stages of neurodegeneration, conforming to the previous reports of a longitudinal progression pattern of dorsal-to-ventral cell death in HD (Vonsattel \& DiFiglia, 1998). More 
specifically, the Map Search findings may suggest that visual attentional processes, relating to the posterior parietal cortex, may specifically be implicated in the visuospatial deficits reported here. However, a more direct and systematic comparison of dorsal versus ventral visual stream involvement, in addition to the possibility of dysfunctional attentional processes, is needed.

We also found grey-matter associations in other cortical regions for Map Search (fusiform and insula) and more broadly for Mental Rotation (superior frontal lobes, fusiform, middle temporal gyrus, temporal pole, hippocampus, parahippocampus, and cingulate). The broader range of regions associated with Mental Rotation, compared to Map Search, is perhaps due to the complex nature of the mental rotation stimuli, which represent 3-dimensional forms, compared to the 2dimensional forms represented on the map used for Map Search. In support, evidence suggests that the basal ganglia sends direct projections to the prefrontal, motor and temporal cortices, and these neural projections are involved in the recognition and registration of complex contextual patterns that are relevant to behaviour (Beiser, Hua, \& Houk, 1997; Lawrence et al., 2000). For example, the fusiform gyrus is involved in colour integration, visuospatial processing and mental imagery (Bogousslavsky, Miklossy, Deruaz, Assal, \& Regli, 1987) which supports its role in both Map Search and Mental Rotation. The broader range of cortical regions implicated in Mental Rotation, such as the frontal and temporal cortices, likely reflects these output projections from the basal ganglia in response to the processing of complex 3-dimensional stimuli.

Both visuospatial tasks showed associations with the fusiform gyrus. The involvement of the fusiform gyrus in colour integration, visuospatial processing and mental imagery (Bogousslavsky et al., 1987) supports its role in both Map Search and Mental Rotation. The finding of temporal lobe involvement in visuospatial deficits in HD does not support the view that the dorsal visual pathway, which does not include the temporal lobes, is specifically implicated in the neuropathology of HD. However, evidence in Alzheimer's disease suggests that both the dorsal and ventral pathways are affected in Alzheimer's disease as a result of both compensatory 
responses and functional reorganisation. In our study, we were unable to tease apart the involvement of the two visual pathways.

\section{Limitations and future studies}

There are key points to consider when interpreting these findings. Firstly, our findings may suggest that tasks involving visual search, or of selective attentional processes, are more sensitive at detecting differences between premanifest HD and controls, than mental rotation. However, it is possible that our measure of mental rotation lacks sufficient sensitivity, or may not engage disease-specific circuits, to detect group differences in the premanifest period, thus, it is impossible to attribute with certainty the lack of findings in the premanifest group to the absence of deficits per se rather than the lack of a sensitive measure. Furthermore, the declines in visual search, that were consistent across all HD groups, may have reflected slowness in processing speed rather than visuospatial deficits. It is therefore possible that a combination of poor visual search ability as well as speed or movement slowness could have contributed to the visual search findings. However, the effects of motor dysfunction on cognitive outcomes are likely to be small (Stout et al., 2011) but remains to be examined specifically for visuospatial tasks. Secondly, we did not adjust our $p$-values in our many comparisons and may have false discoveries in our findings. We were therefore cautious in reporting and interpreting our findings relating to speedaccuracy and longitudinal declines in mental rotation. Thirdly, response time on mental rotation was for correct answers only. We therefore could have failed to detect a true cross-sectional deficits or longitudinal change because some participants may have been over-represented, i.e., those who are naturally better at the tasks or have less disease burden. In addition, the two time points also limits our longitudinal outcomes and more time points should be considered in future studies. Fourthly, we only examined brain volume changes cross-sectionally, making it impossible to make conclusions linking rates of brain deterioration to visuospatial performance. Finally, correlations between posterior brain volume loss and poorer functional capacity cannot be 
interpreted as causal evidence that smaller posterior brain volume cause poorer function. Instead, our correlational findings may reflect a common association between overall HD neuropathology rather than a direct functional association. However, the inclusion of Disease Burden scores in the regression model reduced this overall disease effect and may imply a more specific association. Future research should replicate these findings to more clearly delineate posterior brain functions involving visuospatial tasks in HD. In particular, the current findings should be extended to other possible endpoints of posterior brain dysfunction in HD, such as other cognitive and occulomotor outcomes. In addition, a more comprehensive assessment of visuospatial function is needed, along with longer longitudinal intervals, to fully delineate the multifaceted visuospatial functions in HD.

\section{Acknowledgments}

The authors offer their gratitude to the Track-HD research volunteers, and to their carers and companions who helped make their participation possible. TRACK-HD is supported by the CHDI/High Q Foundation Inc, a not-for-profit organisation dedicated to finding treatments for Huntington's disease. There are no conflicts of interest to report for all authors.

\section{TRACK-HD Investigators}

Canada A Coleman, R Dar Santos (University of British Columbia, Vancouver). France C Jauffret, D Justo (APHP, Hôpital Salpêtriere, Paris). Netherlands E M Dumas, E P ‘t Hart, V

Roedig (Leiden University Medical Centre, Leiden). UK M Say, D Whitehead (University College London, London). USA H Johnson, E Axelson (University of Iowa, Iowa City, IA)

\section{References}

Baizer, J.S., Desimone, R. \& Ungerleider, L.G. (1993). Comparison of subcortical connections of inferior temporal and posterior parietal cortex in monkeys. Vis Neurosci, 10, 59-72. 
Labuschagne-Visuospatial Deficits in Huntington's Disease

Baron-Cohen, S., Wheelwright, S., Hill, J., Raste, Y. \& Plumb, I. (2001). The "Reading the Mind in the Eyes" Test revised version: a study with normal adults, and adults with Asperger syndrome or highfunctioning autism. J Child Psychol Psychiatry, 42, 241-251.

Beiser, D.G., Hua, S.E. \& Houk, J.C. (1997). Network models of the basal ganglia. Curr Opin Neurobiol, 7, 185-190.

Bethel-Fox, C.E. \& Shepard, R. (1988). Mental rotation: effects of stimulus complexity and familiarity. Journal of Experimental Psychology: Human Perception and Performance, 14, 12-23.

Blankenburg, F., Ruff, C.C., Bestmann, S., Bjoertomt, O., Josephs, O., Deichmann, R. \& Driver, J. (2010). Studying the role of human parietal cortex in visuospatial attention with concurrent TMS-fMRI. Cereb Cortex, 20, 2702-2711.

Bogousslavsky, J., Miklossy, J., Deruaz, J.P., Assal, G. \& Regli, F. (1987). Lingual and fusiform gyri in visual processing: a clinico-pathologic study of superior altitudinal hemianopia. $J$ Neurol Neurosurg Psychiatry, 50, 607-614.

Deboer, L.B., Medina, J.L., Davis, M.L., Presnell, K.E., Powers, M.B. \& Smits, J.A. (2013). Associations Between Fear of Negative Evaluation and Eating Pathology During Intervention and 12-Month Follow-up. Cognit Ther Res, 37.

Donner, T.H., Kettermann, A., Diesch, E., Villringer, A. \& Brandt, S.A. (2003). Parietal activation during visual search in the absence of multiple distractors. Neuroreport, 14, 2257-2261.

Duff, K., Beglinger, L.J., Theriault, D., Allison, J. \& Paulsen, J.S. (2010a). Cognitive deficits in Huntington's disease on the Repeatable Battery for the Assessment of Neuropsychological Status. J Clin Exp Neuropsychol, 32, 231-238.

Duff, K., Paulsen, J., Mills, J., Beglinger, L.J., Moser, D.J., Smith, M.M., . . Coordinators of the Huntington Study, G. (2010b). Mild cognitive impairment in prediagnosed Huntington disease. Neurology, 75, 500-507.

Evans, D. \& Rothbart, M.K. (2007). Developing a model for adult temperament. Journal of Research in Personality, 41, 868-888.

Fischl, B. \& Dale, A.M. (2000). Measuring the thickness of the human cerebral cortex from magnetic resonance images. Proc Natl Acad Sci U S A, 97, 11050-11055.

Fischl, B., Salat, D.H., Busa, E., Albert, M., Dieterich, M., Haselgrove, C., . . Dale, A.M. (2002). Whole brain segmentation: automated labeling of neuroanatomical structures in the human brain. Neuron, 33 , 341-355.

Fischl, B., van der Kouwe, A., Destrieux, C., Halgren, E., Segonne, F., Salat, D.H., . . Dale, A.M. (2004). Automatically parcellating the human cerebral cortex. Cereb Cortex, 14, 11-22.

Gomez-Tortosa, E., del Barrio, A., Barroso, T. \& Garcia Ruiz, P.J. (1996). Visual processing disorders in patients with Huntington's disease and asymptomatic carriers. J Neurol, 243, 286-292.

Goodale, M.A. \& Milner, A.D. (1992). Separate visual pathways for perception and action. Trends Neurosci, 15, 20-25.

Hagler, D.J., Jr., Saygin, A.P. \& Sereno, M. (2006). Smoothing and cluster thresholding for cortical surfacebased group analysis of fMRI data. Neuroimage, 33, 1093-1103.

Harris, I.M., Egan, G.F., Sonkkila, C., Tochon-Danguy, H.J., Paxinos, G. \& Watson, J.D. (2000). Selective right parietal lobe activation during mental rotation: a parametric PET study. Brain, 123 ( Pt 1), 65-73.

Harris, I.M., Harris, J.A. \& Caine, D. (2002). Mental-rotation deficits following damage to the right basal ganglia. Neuropsychology, 16, 524-537.

Huber, P.J. (1967). The behavior of maximum likelihood estimates under nonstandard conditions. Proceedings of the Fith Berkeley Symposium on Mathematical Statistics and Probability. Berkeley, CA, University of California Press. 1: 221-233.

HuntingtonStudyGroup (1999). Unified Huntington's Disease Rating Scale-99. Huntington Study Group.

Jacobs, H.I., Van Boxtel, M.P., Heinecke, A., Gronenschild, E.H., Backes, W.H., Ramakers, I.H., . . Verhey, F.R. (2012). Functional integration of parietal lobe activity in early Alzheimer disease. Neurology, $78,352-360$. 
Johnson, E.B., Rees, E.M., Labuschagne, I., Durr, A., Leavitt, B.R., Roos, R.A., . . Scahill, R.I. (2015). The impact of occipital lobe cortical thickness on cognitive task performance: An investigation in Huntington's Disease. Neuropsychologia, 79, 138-146.

Klöppel, S., Gregory, S., Scheller, E., Minkova, L., Razi, A., Durr, A., . . Investigators, T.-O. (2015). Compensation in preclinical Huntington's disease: evidence from the Track-On study. EBioMedicine, 2, 1420-1429.

Kravitz, D.J., Saleem, K.S., Baker, C.I. \& Mishkin, M. (2011). A new neural framework for visuospatial processing. Nat Rev Neurosci, 12, 217-230.

Labuschagne, I., Jones, R., Callaghan, J., Whitehead, D., Dumas, E.M., Say, M.J., . . Investigators, T.-H. (2013). Emotional face recognition deficits and medication effects in pre-manifest through stage-II Huntington's disease. Psychiatry Res, 207, 118-126.

Lawrence, A.D., Watkins, L.H., Sahakian, B.J., Hodges, J.R. \& Robbins, T.W. (2000). Visual object and visuospatial cognition in Huntington's disease: Implications for information processing in corticostriatal circuits. Brain, 123, 1349-1364.

Lineweaver, T.T., Salmon, D.P., Bondi, M.W. \& Corey-Bloom, J. (2005). Differential effects of Alzheimer's disease and Huntington's disease on the performance of mental rotation. J Int Neuropsychol Soc, $11,30-39$.

Mandal, P.K., Joshi, J. \& Saharan, S. (2012). Visuospatial perception: an emerging biomarker for Alzheimer's disease. J Alzheimers Dis, 31 Suppl 3, S117-135.

Mohr, E., Brouwers, P., Claus, J.J., Mann, U.M., Fedio, P. \& Chase, T.N. (1991). Visuospatial cognition in Huntington's disease. Mov Disord, 6, 127-132.

Muller, C.J. \& MacLehose, R.F. (2014). Estimating predicted probabilities from logistic regression: different methods correspond to different target populations. Int J Epidemiol, 43, 962-970.

Murray, L.L. \& Stout, J.C. (1999). Discourse comprehension in Huntington's and Parkinson's diseases. American Journal of Speech-Language Pathology, 8, 137-148.

Oehlert, G.W. (1992). A note on the delta method. The American Statistician, 46, 27-29.

Penney, J.B.J., Vonsattel, J.P., MacDonald, M.E., Gusella, J.F. \& Myers, R.H. (1997). CAG repeat number governs the development rate of pathology in Huntington's disease. Annals of Neurology, 41, 689692.

Perry, R.J. \& Hodges, J.R. (2000). Relationship between functional and neuropsychological performance in early Alzheimer disease. Alzheimer Dis Assoc Disord, 14, 1-10.

Peters, M. \& Battista, C. (2008). Applications of mental rotation figures of the Shepard and Metzler type and description of a mental rotation stimulus library. Brain Cogn, 66, 260-264.

Prvulovic, D., Hubl, D., Sack, A.T., Melillo, L., Maurer, K., Frolich, L., ... Dierks, T. (2002). Functional imaging of visuospatial processing in Alzheimer's disease. Neuroimage, 17, 1403-1414.

Robertson, I.H., Ward, T., Ridgeway, V. \& Nimmo-Smith, I. (1994). The Test of Everday Attention, Bury St. Edmunds: Thames Valley Test Company.

Robins Wahlin, T.B., Lundin, A. \& Dear, K. (2007). Early cognitive deficits in Swedish gene carriers of Huntington's disease. Neuropsychology, 21, 31-44.

Rothman, K.J. (1990). No adjustments are needed for multiple comparisons. Epidemiology, 1, 43-46.

Rubin, L.H., Carter, C.S., Bishop, J.R., Pournajafi-Nazarloo, H., Harris, M.S., Hill, S.K., . . Sweeney, J.A. (2013). Peripheral vasopressin but not oxytocin relates to severity of acute psychosis in women with acutely-ill untreated first-episode psychosis. Schizophr Res, 146, 138-143.

Shepard, R. \& Metzler, J. (1971). Mental rotation of three dimensional objects. Science, 171, 701-703.

Shoulson, I. \& Fahn, S. (1979). Huntington disease: clinical care and evaluation. Neurology, 29, 1-3.

Stout, J.C., Paulsen, J.S., Queller, S., Solomon, A.C., Whitlock, K.B., Campbell, J.C., . . Aylward, E.H. (2011). Neurocognitive signs in prodromal Huntington disease. Neuropsychology, 25, 1-14.

Tabrizi, S.J., Langbehn, D.R., Leavitt, B.R., Roos, R.A., Durr, A., Craufurd, D., . . . investigators, T.-H. (2009). Biological and clinical manifestations of Huntington's disease in the longitudinal TRACK-HD study: cross-sectional analysis of baseline data. Lancet Neurol, 8, 791-801. 
Labuschagne-Visuospatial Deficits in Huntington's Disease

Unschuld, P.G., Edden, R.A., Carass, A., Liu, X., Shanahan, M., Wang, X., . . Ross, C.A. (2012). Brain metabolite alterations and cognitive dysfunction in early Huntington's disease. Mov Disord, 27, 895-902.

Yeterian, E.H. \& Pandya, D.N. (1995). Corticostriatal connections of extrastriate visual areas in rhesus monkeys. J Comp Neurol, 352, 436-457. 


\section{Tables}

Table 1. Participant demographics as measured at baseline

\begin{tabular}{|c|c|c|c|c|c|}
\hline & Controls & PreA & PreB & HD1 & HD2 \\
\hline \multicolumn{6}{|l|}{ Demographics: } \\
\hline Number of participants ${ }^{\mathrm{a}}$ & 110 & 55 & 64 & 59 & 45 \\
\hline Female $(\%)$ & $62(56 \%)$ & $33(60 \%)$ & $34(53 \%)$ & $38(64 \%)$ & $19(42 \%)$ \\
\hline \multicolumn{6}{|l|}{ Education $(\%)^{\mathrm{b}}$} \\
\hline Level 1 & $1(1 \%)$ & 0 & 0 & $1(2 \%)$ & 0 \\
\hline Level 2 & $20(18 \%)$ & $4(7 \%)$ & $7(11 \%)$ & $11(19 \%)$ & $12(27 \%)$ \\
\hline Level 3 & $12(11 \%)$ & $11(20 \%)$ & $13(20 \%)$ & $14(24 \%)$ & $14(31 \%)$ \\
\hline Level 4 & $32(30 \%)$ & $14(25 \%)$ & $19(30 \%)$ & $9(15 \%)$ & $8(18 \%)$ \\
\hline Level 5 & $30(27 \%)$ & $22(40 \%)$ & $20(31 \%)$ & $18(31 \%)$ & $10(22 \%)$ \\
\hline Level 6 & $15(14 \%)$ & $4(7 \%)$ & $5(8 \%)$ & $6(10 \%)$ & $1(2 \%)$ \\
\hline Age, mean years (SD) & $48.2(10.2)$ & $41.0(9.7)$ & $43.0(8.7)$ & $48.4(10.2)$ & $51.3(9.7)$ \\
\hline CAG repeat length, mean (SD) & - & $42.3(2.1)$ & $43.8(2.3)$ & $43.7(2.9)$ & $43.7(3.4)$ \\
\hline Disease Burden Score, mean (SD) & - & $259.7(29.0)$ & $339.7(39.3)$ & $371.8(75.5)$ & $394.9(84.5)$ \\
\hline Total Motor Score, mean $(\mathrm{SD})^{\mathrm{c}}$ & $1.4(1.9)$ & $3.4(2.9)$ & $5.0(3.6)$ & $21.5(9.8)$ & $33.8(11.3)$ \\
\hline Total Functional Capacity, mean (SD) & $13.0(0.2)$ & $12.9(0.6)$ & $12.8(0.7)$ & $12.1(0.9)$ & $8.6(1.1)$ \\
\hline
\end{tabular}

PreA = premanifest HD far from diagnosis; PreB = premanifest HD near diagnosis; HD1 = HD stage-1; $\mathrm{HD} 2=\mathrm{HD}$ stage- $2 ; \mathrm{SD}=$ standard deviation; $\%=$ percentage .

a. All participants who attended either baseline or 1-year follow-up.

b. Education level was reported according to the International Standard Classification of Education.

c. Total motor and functional capacity scores were measured using the UHDRS (Huntington Study Group, 1999). 
Table 2. Between-group contrasts for cross-sectional (baseline) differences and annualised change differences on the Map Search and Mental Rotation tasks.

\begin{tabular}{|c|c|c|c|c|c|c|c|c|}
\hline & PreA vs. Controls & $p$ & PreB vs. Controls & $p$ & HD1 vs. Controls & $p$ & HD2 vs. Controls & $p$ \\
\hline \multicolumn{9}{|c|}{ Map Search (no.correct) $^{\mathrm{a}}$} \\
\hline \multicolumn{9}{|l|}{ Baseline } \\
\hline $1 \mathrm{~min}$ & $-6.3(-10.1,-2.5)$ & 0.0011 & $-8.8(-12.5,-5.0)$ & $<0.0001$ & $-14.8(-18.3,-11.4)$ & $<0.0001$ & $-22.7(-26.2,-19.1)$ & $<0.0001$ \\
\hline $2 \min$ & $-3.3(-6.7,0.1)$ & 0.0603 & $-6.9(-10.3,-3.5)$ & $<0.0001$ & $-13.9(-17.8,-10.0)$ & $<0.0001$ & $-26.9(-31.4,-22.4)$ & $<0.0001$ \\
\hline \multicolumn{9}{|l|}{ Annualised change } \\
\hline $1 \mathrm{~min}$ & $1.1(-2.1,4.2)$ & 0.5145 & $-1.8(-4.4,0.8)$ & 0.1747 & $-2.0(-4.6,0.7)$ & 0.1473 & $-3.4(-5.8,-1.1)$ & 0.0043 \\
\hline $2 \min$ & $0.6(-2.1,3.3)$ & 0.6650 & $0.2(-2.4,2.7)$ & 0.9062 & $-0.6(-3.0,1.8)$ & 0.6231 & $-3.8(-7.0,-0.7)$ & 0.0167 \\
\hline \multicolumn{9}{|c|}{ Mental Rotation (\% correct) ${ }^{b}$} \\
\hline \multicolumn{9}{|l|}{ Baseline } \\
\hline SAME $65^{\circ}$ & $-0.009(-0.078,0.060)$ & 0.8000 & $-0.035(-0.101,0.031)$ & 0.2985 & $-0.044(-0.112,0.024)$ & 0.2087 & $-0.181(-0.279,-0.084)$ & 0.0003 \\
\hline SAME $125^{\circ}$ & $0.021(-0.066,0.108)$ & 0.6309 & $0.026(-0.047,0.098)$ & 0.4869 & $-0.052(-0.126,0.022)$ & 0.1657 & $-0.140(-0.222,-0.058)$ & 0.0008 \\
\hline MIRROR $65^{\circ}$ & $-0.060(-0.157,0.038)$ & 0.2294 & $-0.042(-0.117,0.034)$ & 0.2827 & $-0.170(-0.250,-0.090)$ & $<0.0001$ & $-0.154(-0.255,-0.054)$ & 0.0027 \\
\hline MIRROR $125^{\circ}$ & $0.007(-0.083,0.096)$ & 0.8859 & $-0.047(-0.123,0.029)$ & 0.2223 & $-0.175(-0.257,-0.093)$ & $<0.0001$ & $-0.110(-0.207,-0.013)$ & 0.0260 \\
\hline \multicolumn{9}{|l|}{ Annualised change } \\
\hline SAME $65^{\circ}$ & $0.022(-0.062,0.107)$ & 0.6025 & $-0.024(-0.087,0.040)$ & 0.4675 & $-0.095(-0.175,-0.015)$ & 0.0204 & $-0.022(-0.112,0.068)$ & 0.6274 \\
\hline SAME $125^{\circ}$ & $-0.055(-0.155,0.045)$ & 0.2798 & $-0.050(-0.133,0.034)$ & 0.2441 & $-0.136(-0.238,-0.035)$ & 0.0085 & $-0.036(-0.148,0.075)$ & 0.5222 \\
\hline MIRROR $65^{\circ}$ & $0.097(0.003,0.191)$ & 0.0431 & $-0.030(-0.121,0.061)$ & 0.5228 & $0.084(-0.009,0.177)$ & 0.0773 & $-0.051(-0.172,0.069)$ & 0.4040 \\
\hline
\end{tabular}




\begin{tabular}{|c|c|c|c|c|c|c|c|c|}
\hline MIRROR $125^{\circ}$ & $-0.076(-0.163,0.011)$ & 0.0855 & $-0.044(-0.130,0.042)$ & 0.3190 & $-0.037(-0.131,0.057)$ & 0.4406 & $-0.132(-0.235,-0.028)$ & 0.0127 \\
\hline \multicolumn{9}{|c|}{ Mental Rotation (response time) } \\
\hline \multicolumn{9}{|l|}{ Baseline } \\
\hline SAME $65^{\circ}$ & $322(-188,832)$ & 0.2162 & $937(451,1,424)$ & 0.0002 & $630(-40,1,301)$ & 0.0655 & $675(-114,1,464)$ & 0.0936 \\
\hline SAME $125^{\circ}$ & $242(-377,862)$ & 0.4431 & $1,020(343,1,698)$ & 0.0032 & $129(-727,984)$ & 0.7683 & $680(-310,1,669)$ & 0.1781 \\
\hline MIRROR $65^{\circ}$ & $43(-610,696)$ & 0.8970 & $1,000(387,1,613)$ & 0.0014 & $83(-620,785)$ & 0.8179 & $461(-441,1,363)$ & 0.3163 \\
\hline MIRROR $125^{\circ}$ & $246(-424,915)$ & 0.4720 & $972(369,1,576)$ & 0.0016 & $39(-826,905)$ & 0.9288 & $98(-869,1,065)$ & 0.8428 \\
\hline \multicolumn{9}{|l|}{ Annualised change } \\
\hline SAME $65^{\circ}$ & $133(-417,682)$ & 0.6362 & $-515(-1,082,51)$ & 0.0746 & $30(-600,660)$ & 0.9254 & $227(-632,1,085)$ & 0.6050 \\
\hline SAME $125^{\circ}$ & $216(-464,896)$ & 0.5342 & $-431(-1,222,360)$ & 0.2857 & $-32(-828,763)$ & 0.9367 & $-592(-1,652,469)$ & 0.2741 \\
\hline MIRROR $65^{\circ}$ & $795(-11,1,601)$ & 0.0532 & $-336(-1,031,359)$ & 0.3431 & $483(-222,1,189)$ & 0.1792 & $-157(-994,680)$ & 0.7136 \\
\hline MIRROR $125^{\circ}$ & $187(-551,925)$ & 0.6194 & $-128(-810,553)$ & 0.7120 & $61(-924,1,045)$ & 0.9040 & $-13(-976,950)$ & 0.9790 \\
\hline
\end{tabular}

Note. Contrasts are means (95\% confidence interval) and are standardised to the marginal distribution of age, sex, education and site in the groups combined.

PreA = premanifest HD far from diagnosis; PreB = premanifest HD near diagnosis; HD1 = HD stage-1; HD2 = HD stage-2. $p=$ significance $\mathrm{p}$-value

a. Map Search is presented as the number of objects correctly identified

b. Mental Rotation percent correct

c. Mental Rotation response time in milliseconds 
Labuschagne-Visuospatial deficits in Huntington's Disease

Table 3. Partial correlations between disease severity measures and outcomes at baseline.

$\begin{array}{lllllllll}\text { PreA } & p & \text { PreB } & p & \text { HD1 } & p & \text { HD2 } & p & \begin{array}{c}\text { Early } \\ \text { HD }\end{array}\end{array}$

\begin{tabular}{|c|c|c|c|c|c|c|c|c|c|c|}
\hline \multicolumn{11}{|l|}{ Map Search: } \\
\hline Disease Burden Score & 0.22 & 0.1338 & 0.05 & 0.7205 & -0.31 & 0.0321 & -0.11 & 0.5032 & & \\
\hline Total Motor Score & & & & & -0.30 & 0.0406 & -0.37 & 0.0233 & & \\
\hline $\begin{array}{l}\text { Total Functional } \\
\text { Capacity }\end{array}$ & & & & & & & & & 0.38 & 0.0002 \\
\hline \multicolumn{11}{|c|}{ Mental Rotation - SAME: } \\
\hline Disease Burden Score & -0.11 & 0.4744 & -0.22 & 0.1009 & -0.04 & 0.7708 & -0.04 & 0.8036 & & \\
\hline Total Motor Score & & & & & 0.06 & 0.6576 & -0.35 & 0.0337 & & \\
\hline $\begin{array}{l}\text { Total Functional } \\
\text { Capacity }\end{array}$ & & & & & & & & & 0.29 & 0.0047 \\
\hline \multicolumn{11}{|c|}{ Mental Rotation - MIRROR: } \\
\hline Disease Burden Score & 0.12 & 0.4388 & -0.24 & 0.0759 & 0.03 & 0.8092 & -0.07 & 0.6787 & & \\
\hline Total Motor Score & & & & & -0.10 & 0.4876 & -0.19 & 0.2513 & & \\
\hline $\begin{array}{l}\text { Total Functional } \\
\text { Capacity }\end{array}$ & & & & & & & & & -0.11 & 0.2796 \\
\hline
\end{tabular}

Note. Correlations are adjusted for age, CAG length, sex, education and site. For Map Search, we used the 1minute outcome, and for Mental Rotation we used average accuracy at 65 and 125 degrees in both SAME and MIRROR conditions. Since Total Functional Capacity score is the defining difference between disease stages, outcome correlations with this measure are reported across both stages combined. PreA $=$ premanifest HD far from diagnosis; PreB = premanifes HD near diagnosis; HD1 = HD stage-1; HD2 = HD stage-2; $\mathrm{P}=$ significance $\mathrm{p}$-value. Disease Burden Scores are calculated as age x [CAG-35.5] (Penney, et al., 1995). Total Motor Score and Total Functioning Capacity are calculated from the UHDRS (Huntington Study Group, 1999). 


\section{Figure legends}

Figure 1. Mean Mental Rotation accuracy (A) and response time (B) at baseline (top, all degrees of rotation) and annualised change (bottom, 65 and 125 degrees). Means are standardised to the marginal distribution of age, sex, education and site in the groups combined. $\mathrm{Ctrl}=$ controls; $\mathrm{Pre} \mathrm{A}=$ premanifest $\mathrm{HD}$ far from diagnosis; PreB = premanifes HD near diagnosis; HD1 = HD stage-1; HD2 = HD stage-2.

Figure 2. Mean Map Search 1- and 2-minute scores at baseline and at annualised follow up, standardised to the marginal distribution of age, sex, education and site in the groups combined. $\mathrm{Ctrl}=$ controls; PreA $=$ premanifest HD far from diagnosis; PreB = premanifes HD near diagnosis; HD1 = HD stage-1; HD2 = HD stage-2.

Figure 3. Mean Mental Rotation response time at baseline as a function of accuracy at 65 and 125 degrees under both SAME and MIRROR conditions. Means are standardised to the marginal distribution of age, sex, education and site in the groups combined. $\mathrm{Ctrl}=$ controls; PreA = premanifest HD far from diagnosis; PreB = premanifes HD near diagnosis; HD1 = HD stage-1; HD2 = HD stage-2.

Figure 4. Structural brain correlations to visuospatial performance at baseline. Correlations included all the gene-positive groups combined. For Map Search, we used the 1-minute scores, and for Mental Rotation we used accuracy for 65 and 125 degrees combined. Voxel based morphometry results for grey-matter (A) and white-matter (B) are corrected for multiple comparisons using the false discovery rate at $\mathrm{P}<0.05$, and cortical thickness results (C) are corrected using a Monte Carlo cluster-wise correction at $\mathrm{P}<0.05$. Voxel based morphometry associations were adjusted for age, gender, site, education, intracranial volume, CAG and disease burden, and cortical thickness associations were adjusted for age, gender, site, education, CAG and disease burden. $\mathrm{L}=$ left; $\mathrm{R}=$ right. 
A
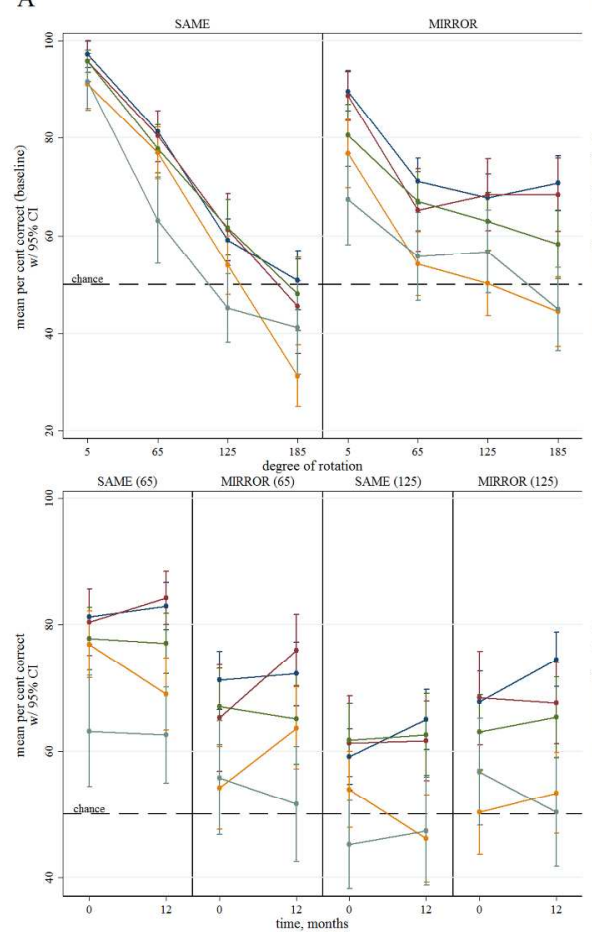

B
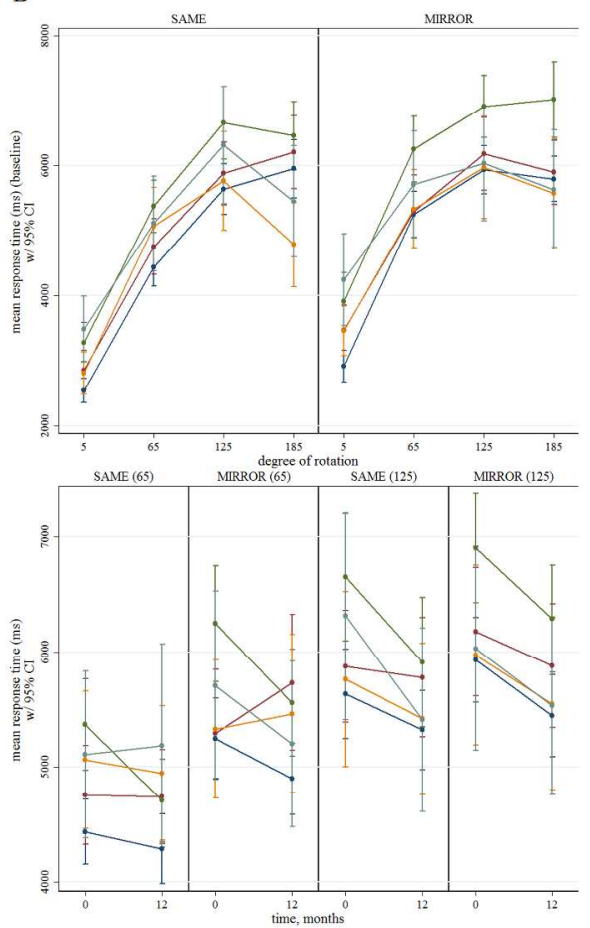

Figure 1

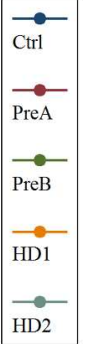

Figure 1. Mean Mental Rotation accuracy (A) and response time (B) at baseline (top, all degrees of rotation) and annualised change (bottom, 65 and 125 degrees). Means are standardised to the marginal distribution of age, sex, education and site in the groups combined. Ctrl = controls; PreA = premanifest HD far from diagnosis; PreB = premanifes HD near diagnosis; HD1 = HD stage-1; HD2 = HD stage- 2 .

$783 \times 569 \mathrm{~mm}(72 \times 72 \mathrm{DPI})$ 


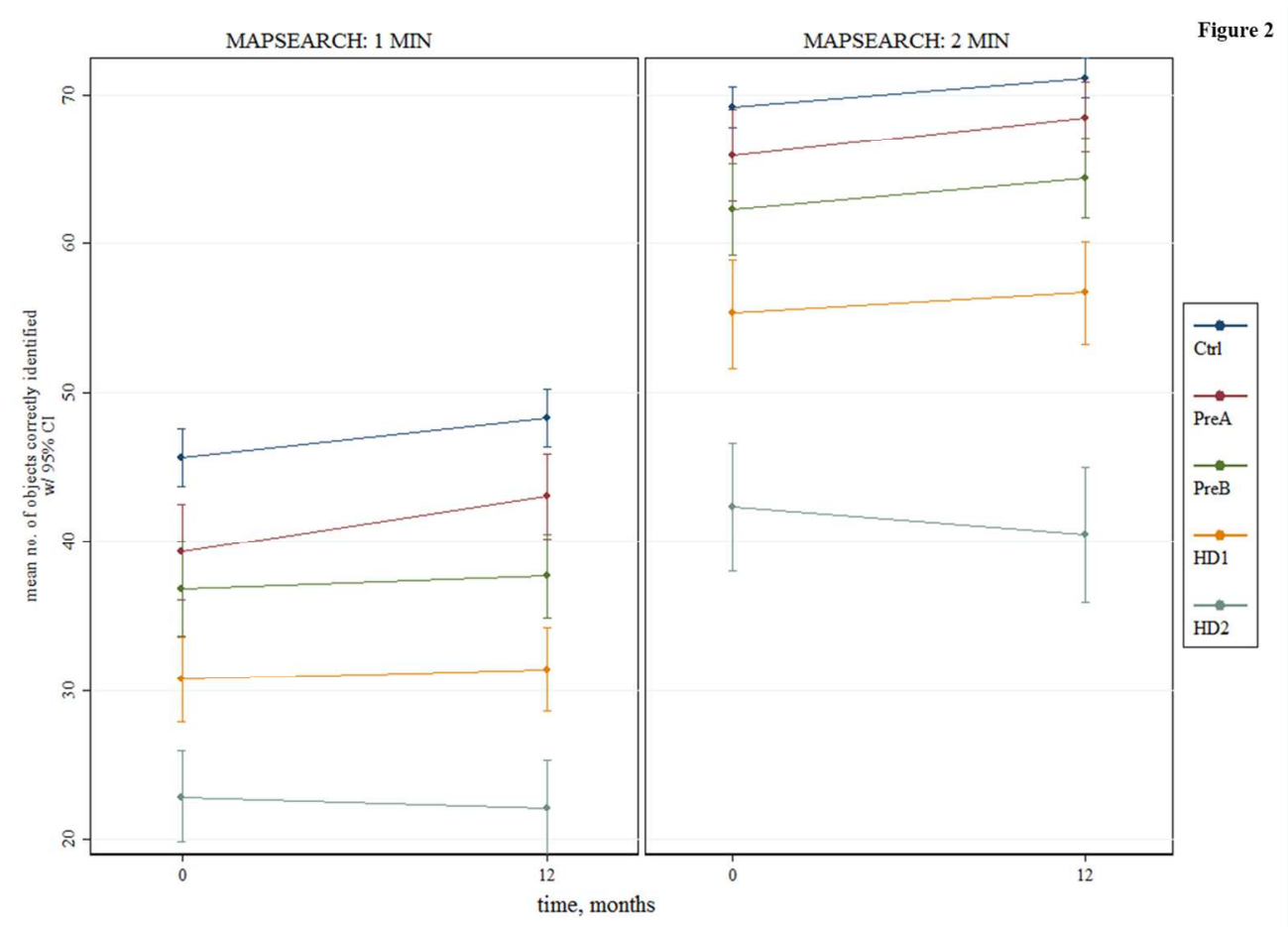

Figure 2. Mean Map Search 1- and 2-minute scores at baseline and at annualised follow up, standardised to the marginal distribution of age, sex, education and site in the groups combined. Ctrl = controls; PreA = premanifest HD far from diagnosis; PreB = premanifes HD near diagnosis; HD1 = HD stage-1; HD2 = HD stage- 2 .

$381 \times 276 \mathrm{~mm}(100 \times 100 \mathrm{DPI})$ 


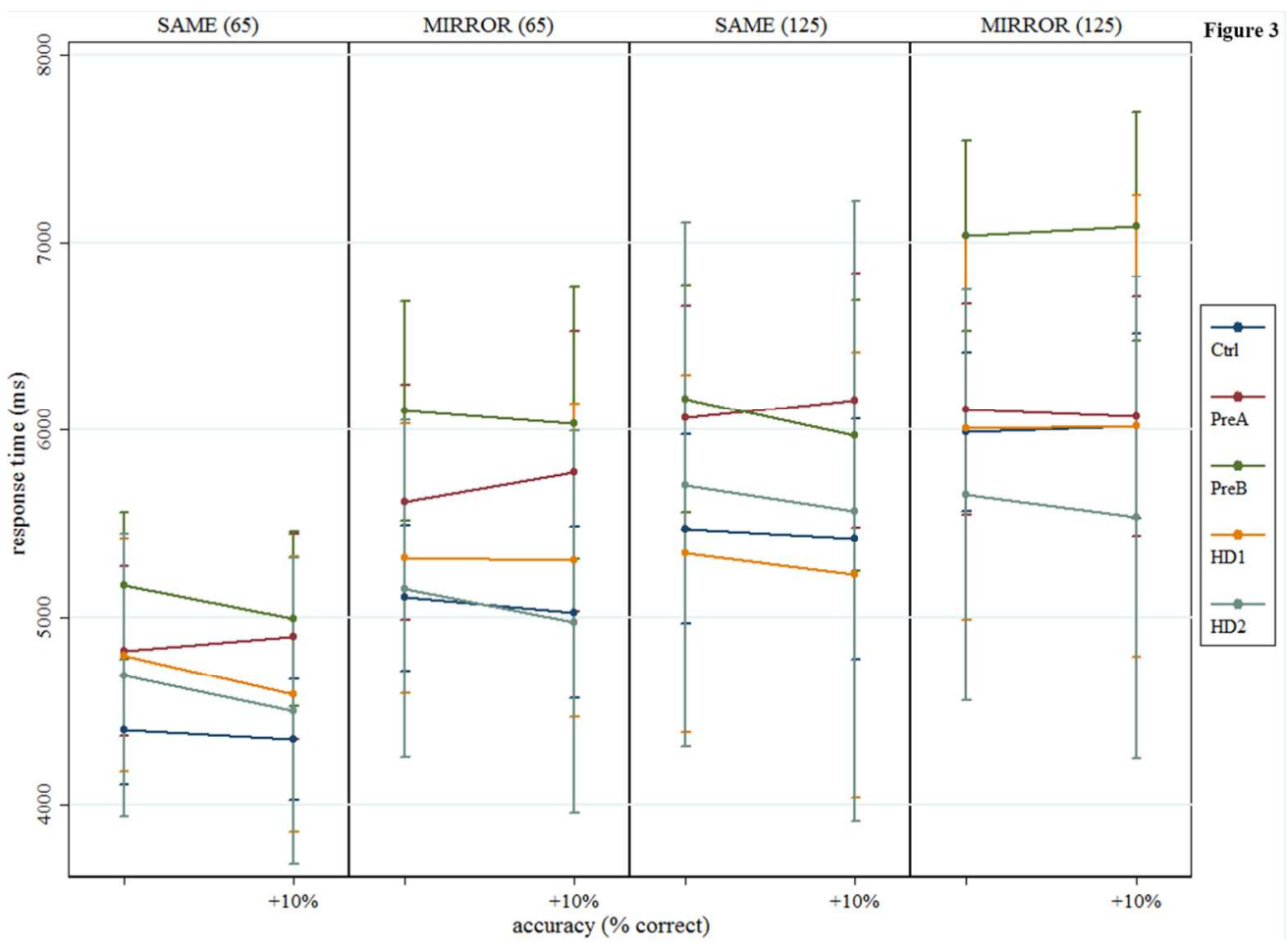

Figure 3. Mean Mental Rotation response time at baseline as a function of accuracy at 65 and 125 degrees under both SAME and MIRROR conditions. Means are standardised to the marginal distribution of age, sex, education and site in the groups combined. Ctrl = controls; PreA = premanifest HD far from diagnosis; PreB = premanifes HD near diagnosis; HD1 = HD stage-1; HD2 = HD stage- 2 . $381 \times 276 \mathrm{~mm}(100 \times 100 \mathrm{DPI})$ 


\section{Supplementary material}

Supplementary Table 1 Between-group contrasts for cross-sectional (baseline) differences in speed-accuracy tradeoff in the Mental Rotation task. Tradeoff is defined as extra time taken (ms) to respond correctly per $10 \%$ absolute increase in overall accuracy. Contrasts are means (95\% confidence intervals) and are adjusted for age, sex, education and site.

\begin{tabular}{cccccccccc}
\hline & PreA vs. Control & P & PreB vs. Control & P & HD1 vs. Control & P & HD2 vs. Control & P \\
\hline SAME: & & & & & & & \\
65 & $132(-94,357)$ & 0.2522 & $-126(-331,78)$ & 0.2249 & $-160(-413,93)$ & 0.2141 & $-140(-322,42)$ & 0.1305 \\
125 & $145(-73,363)$ & 0.1929 & $-144(-405,117)$ & 0.2790 & $-61(-402,280)$ & 0.7245 & $-88(-447,271)$ & 0.6308 \\
MIRROR: & & & & & & & \\
65 & $239(3,476)$ & 0.0476 & $10(-241,260)$ & 0.9388 & $69(-146,284)$ & 0.5287 & $-103(-351,145)$ & 0.4165 \\
125 & $-66(-280,148)$ & 0.5454 & $20(-187,228)$ & 0.8467 & $-19(-311,273)$ & 0.8990 & $-149(-451,154)$ & 0.3353
\end{tabular}

PreA = premanifest Huntington's disease far from diagnosis; PreB = premanifest Huntington's disease near diagnosis; HD1 = Huntington's disease stage-1; HD2 = Huntington's disease stage-2; $\mathrm{P}=$ significance $\mathrm{p}$-value. 